\title{
Synthesis and Antimicrobial Activity of Novel Series of Diversely Substituted Acetyl Pyrazoline Bearing Biphenyl Carbonitrile Motif
}

\author{
Pineshkumar N. Patel ${ }^{1 *}$, Denish C. Karia ${ }^{2}$ \\ ${ }^{1}$ Research Scholar, Kadi SarvaVishwavidhyalaya Gandhinagar, Gujarat, India \\ 2Department of Chemistry, Patel J D K Davolwala Science College, Borsad, Gujarat, India \\ *1ppinesh41891@gmail.com, 2denishkaria@yahoo.com
}

\section{Keywords: Acetyl Pyrazoline, Biphenyl carbonitrile, Antimicrobial, Antifungal.}

\begin{abstract}
Novel series of diversely substituted acetyl pyrazoline having biphenyl carbonitrile motif have been synthesized. The reaction of 2-cyno-4'-bromomethyl biphenyl with1-(4-hydroxyphenyl)-ethanone resulted in acetophenone derivative of biphenyl-2-carbonitrile. This acetophenone derivative was condensed with substituted aromatic aldehyde in mixed solvent resulted in various substituted chalcones. These chalcones were further cyclized using hydrazine hydrate in presence of glacial acetic acid to produce titled compound derivatives. The chemical structures of synthesized compounds were elucidated by ${ }^{1} \mathrm{H}-\mathrm{NMR},{ }^{13} \mathrm{C}-\mathrm{NMR}$ FT-IR and mass spectra. Synthesized compounds were screened for their antimicrobial activity by broth dilution method. Out of twelve newly synthesized compounds, eight compounds are found to be equipotent to Ampicillin.
\end{abstract}

\section{Introduction:}

Medicinally important heterocyclic compounds are widely distributed in the nature and known to mankind since early of 18 th century. After the $2^{\text {nd }}$ world war, significant efforts were made to develop novel drugs with improved efficiency and to fight against new diseases. Among all, pyrazoline is one of the important classes of heterocyclic compound possessing various therapeutic values. Pyrazoline is a five member ring with two adjacent Nitrogen atoms in a ring. Many synthetic medicinal compound possessed pyrazoline motif functionalized with various groups. Besides this, significant researches have been continued on pyrazoline derivatives around the world. Pyrazoline compounds are acquiring more importance because of their immense biological and pharmacological potential. This class of compound possesses wide spectrum of biological activities such as antimicrobial $[1,2,3]$, anti-inflammatory and analgesic [4,5], anticancer [6,7], antidepressant [8], anti-tuberculosis [9,10], antiamoebic [11] antioxidant [12,13], antihyperglycemic [14], anti-epileptic[15,16], anti-parkinson and anti-alzheimer[17].

Though significant research efforts have been made to develop drugs which can be used to cure diseases born out of microbial infections, still it is required to develop novel drugs with improved efficiency against resistant microbes $[18,19]$. Literature survey reveals that the pyrazoline class compounds possesses various therapeutic properties and can be further explored to have better drug molecule. Such numerous biological and medicinal values of pyrazoline compounds prompted us to develop novel acetyl pyrazoline molecules having biphenyl carbonitrile.

In the present work, novel series of acetyl pyrazoline functionalized with biphenyl carbonitrile have been synthesized by reaction of chalcon with hydrazine hydrate in the presence of acetic acid. The easy work up and purification methods produced almost pure compounds. All synthesized compounds were screened for their potential antibacterial and antifungal activity using standard drugs and stains. 


\section{Materials and Method:}

\section{Materials:}

All key raw materials, reagents and solvents were of commercial grade and used after further purification. All melting points were measured using open capillaries in a liquid paraffin bath and were uncorrected. The completion of reaction was monitored by thin layer chromatography using silica gel-G as absorbent and Toluene: Ethyl acetate was employed as mobile phase. The visualization of TLC was accomplished by UV light and Iodine. IR spectra (KBr pallet) were recorded on FT-IR, Perkin Elmer RX1 spectrophotometer and NMR spectra on BRUKER AVANCE II (400 MHz) using TMS as internal standard (chemical shifts in $\delta$ ppm).

\section{Methods:}

In the present work, novel acetyl pyrazoline derivatives were prepared by following general reaction scheme as shown in figure 1.1. The physical constants of synthesized compounds are mentioned in Table 1.1. Synthesized compounds were screened for antibacterial and antifungal activity. The results of antibacterial and antifungal activity are depicted in Table 1.2 and Table 1.3 respectively.

\section{Preparation of 4'-(4-Acetyl-phenoxymethyl)-biphenyl-2-carbonitrile (AC-1):}

A mixture of 2-cyno-4'-bromomethyl-biphenyl (0.037 moles), 4'-hydroxy acetophenone $(0.040$ moles $)$ and sodium carbonate $(0.074$ moles $)$ in dimethyl formamide $(20 \mathrm{ml})$ was heated at $75-80^{\circ} \mathrm{C}$ for 4 hours. After completion of reaction, the mass was cooled to $30^{\circ} \mathrm{C}$ and drawn in water. The resultant precipitates were filtered and dried. The crude product was purified in methanol to isolate 4'-(4-acetyl-phenoxymethyl)-biphenyl-2-carbonitrile as pure, white crystalline powder. The yield of this step was $83 \%$.

Preparation of 4'-\{4-[3-(4-Chloro-phenyl)-acryloyl]-phenoxymethyl\}-biphenyl-2-carbonitrile (AC-2, 1-12) [20]:

AC-1 (1 g, 3.05 mmoles) and substituted aromatic aldehyde $(3.11$ mmoles $)$ were dissolved in a binary mixture of dimethyl formamide and methanol $(1: 1)$. The solution was cooled to $25^{\circ} \mathrm{C}$ and added 50\% sodium hydroxide ( $0.48 \mathrm{~g}, 6 \mathrm{mmoles})$. Then, the reaction mass was stirred at $25-$ $30^{\circ} \mathrm{C}$ for 24 hours. After completion of the reaction, the mass was drawn in water and $\mathrm{pH}$ was adjusted to 2 using 16\% hydrochloric acid. Resultant solids were filtered, washed with water and was further purified in methanol. The yield of this step was $80 \%$.

\section{Preparation of 4'-\{4-[1-Acetyl-5-(4-chloro-phenyl)-4,5-dihydro-1H-pyrazol-3-yl]- phenoxymethyl $\}$ - biphenyl-2-carbonitrile (APC, 1-12):}

To a mixture of AC-2 (2.0 mmoles) in glacial acetic acid, hydrazine hydrate (10.0 mmoles) was added and the mass was heated to reflux for 6 hours. After completion of reaction, the mass was drawn in water. The precipitated solids were filtered, washed with water and dried. This crude product was dissolved in toluene, treated with carbon and further purified using mixture of ethyl acetate and diethyl ether to obtain white powder. The yield of this step was $56 \%$, m.p.: $159-160^{\circ} \mathrm{C}$.

\section{Representative Spectral Data:}

4'-\{4-[1-Acetyl-5-(4-chloro-phenyl)-4,5-dihydro-1H-pyrazol-3-yl]-phenoxymethyl\}-biphenyl-2carbonitrile (APC-1)

${ }^{1} \mathrm{H}$ NMR (DMSO) $\delta$ ppm: $2.31\left(3 \mathrm{H}, \mathrm{s},-\mathrm{CH}_{3}\right), 3.11-3.84\left(2 \mathrm{H}, \mathrm{d},-\mathrm{CH}_{2^{-}}\right.$, heterocyclic), 5.24 $\left(2 \mathrm{H}, \mathrm{s},-\mathrm{CH}_{2}-\mathrm{O}-\right), 5.50\left(1 \mathrm{H}, \mathrm{d},-\mathrm{CH}\right.$, heterocyclic), 7.09-8.18 (16H, m, Ar-H). ${ }^{13} \mathrm{C}$ NMR (DMSO) $\delta$ ppm: $21.57\left(-\underline{\mathrm{CH}}_{3}-\mathrm{C}=\mathrm{O}\right), 39.8\left(-\underline{\mathrm{CH}}_{2^{-}}\right.$, heterocyclic), 41.95 ( $>\underline{\mathrm{CH}}-$, heterocyclic), $78.71\left(-\underline{\mathrm{CH}}_{2}-\mathrm{O}\right)$, $110.30(-\underline{\mathrm{C}} \equiv \mathrm{N}), 114.84-144.12$ (Aromatic $-\mathrm{C}), 153.5$ ( $>\underline{\mathrm{C}}<$, heterocyclic), 159.92 (-O-Aromatic $\underline{\mathrm{C}}$ ), 167.23(- $\left.-\mathrm{CH}_{3}-\mathrm{C}=\mathrm{O}\right)$. FT-IR, $\left(\mathrm{KBr}, \mathrm{cm}^{-1}\right): 760.14$ (o-substituted Benzene), 828 (p-Substituted Benzene), 1032 (Ar-Cl), 1175 (-C-O-, ether), $1604(-\mathrm{C}=\mathrm{N}$, heterocyclic), $1657 \& 1259$ ( $>\mathrm{C}=\mathrm{O}$ of $\left.\mathrm{COCH}_{3}\right), 2223.30(\mathrm{C} \equiv \mathrm{N}), 2229 \& 1361\left(-\mathrm{CH} 2-\right.$, of ether). Mass: $506.16\left(\mathrm{M}^{+}+1\right)$. 


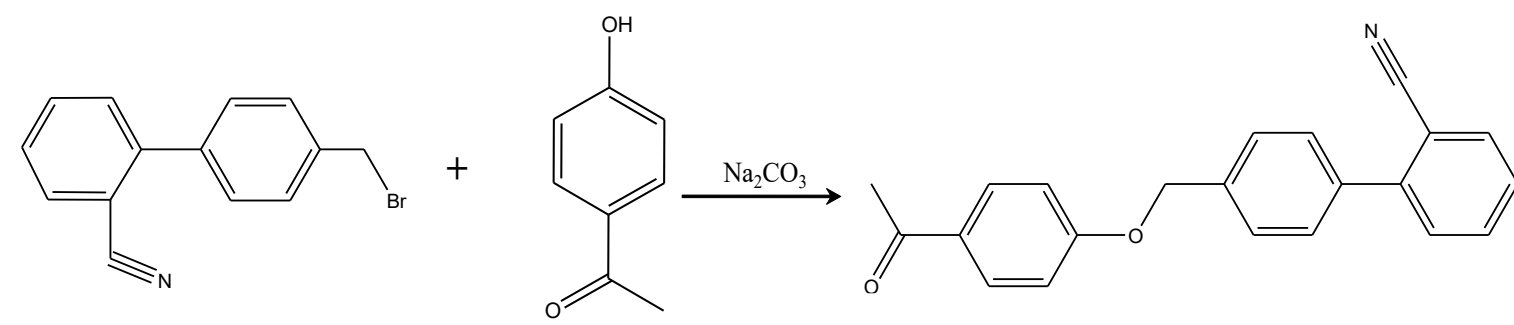

4'-Bromomethyl-biphenyl-2-carbonitrile

4'-(4-Acetyl-phenoxymethyl)-biphenyl-2-carbonitrile

AC-1

$\mathrm{AC}-1+$

Aldehyde
AC-2 $+\mathrm{N}_{2} \mathrm{H}_{4} \cdot \mathrm{H}_{2} \mathrm{O}$

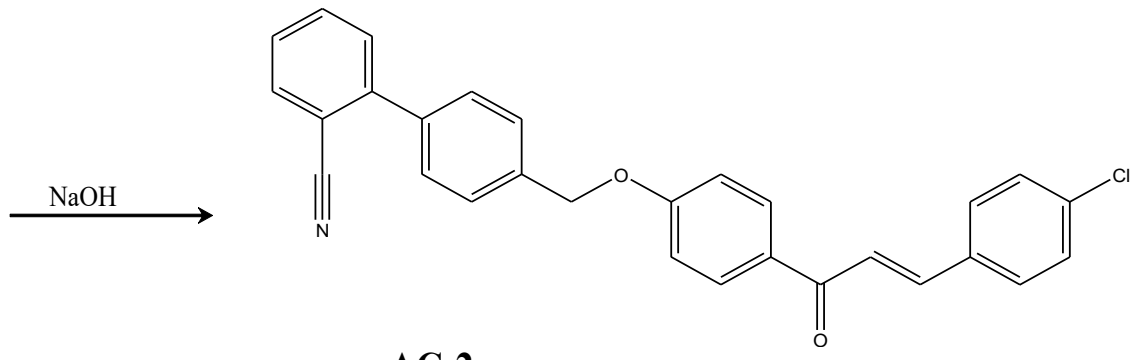

AC-2

4'-\{4-[3-(4-Chloro-phenyl)-acryloyl]-phenoxymethyl $\}$ biphenyl-2-carbonitrile

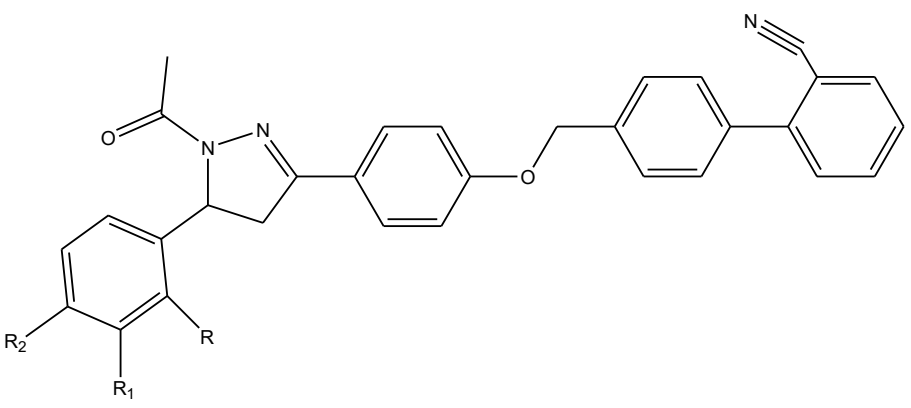

4'-\{4-[1-Acetyl-5-(4-chloro-phenyl)-4,5-dihydro-1H-pyrazol-3yl]-phenoxymethyl\}-biphenyl-2-carbonitrile

APC, 1-12

Figure 1.1: General Synthesis scheme 
Table 1.1: Physical constants of synthesized compounds

\begin{tabular}{|c|c|c|c|c|c|c|c|c|}
\hline Sr.No & Name & $\mathbf{R}$ & $\mathbf{R}_{\mathbf{1}}$ & $\mathbf{R}_{\mathbf{2}}$ & $\begin{array}{c}\text { Molecular } \\
\text { Formula }\end{array}$ & $\begin{array}{c}\text { Mol. } \\
\text { Weight }\end{array}$ & $\begin{array}{c}\text { Melting } \\
\text { Point } \\
{\left[{ }^{\circ} \mathbf{C}\right]}\end{array}$ & $\begin{array}{c}\text { Rf } \\
\text { Value }\end{array}$ \\
\hline 1 & APC-1 & $\mathrm{H}$ & $\mathrm{H}$ & $\mathrm{Cl}$ & $\mathrm{C}_{31} \mathrm{H}_{24} \mathrm{ClN}_{3} \mathrm{O}_{2}$ & 505.99 & 159 & 0.55 \\
\hline 2 & APC-2 & $\mathrm{Cl}$ & $\mathrm{H}$ & $\mathrm{H}$ & $\mathrm{C}_{31} \mathrm{H}_{24} \mathrm{ClN}_{3} \mathrm{O}_{2}$ & 505.99 & 105 & 0.64 \\
\hline 3 & APC-3 & $\mathrm{H}$ & $\mathrm{Cl}$ & $\mathrm{H}$ & $\mathrm{C}_{31} \mathrm{H}_{24} \mathrm{ClN}_{3} \mathrm{O}_{2}$ & 505.99 & 126 & 0.60 \\
\hline 4 & APC-4 & $\mathrm{Br}$ & $\mathrm{H}$ & $\mathrm{H}$ & $\mathrm{C}_{31} \mathrm{H}_{24} \mathrm{BrN}_{3} \mathrm{O}_{2}$ & 550.45 & 160 & 0.60 \\
\hline 5 & APC-5 & $\mathrm{H}$ & $\mathrm{Br}$ & $\mathrm{H}$ & $\mathrm{C}_{31} \mathrm{H}_{24} \mathrm{BrN}_{3} \mathrm{O}_{2}$ & 550.45 & 128 & 0.64 \\
\hline 6 & APC-6 & $\mathrm{H}$ & $\mathrm{H}$ & $\mathrm{H}$ & $\mathrm{C}_{31} \mathrm{H}_{25} \mathrm{~N}_{3} \mathrm{O}_{2}$ & 471.55 & $101-103$ & 0.65 \\
\hline 7 & APC-7 & $\mathrm{H}$ & $\mathrm{H}$ & $\mathrm{CH}_{3}$ & $\mathrm{C}_{32} \mathrm{H}_{27} \mathrm{~N}_{3} \mathrm{O}_{2}$ & 485.58 & 136 & 0.58 \\
\hline 8 & APC-8 & $\mathrm{CH}$ & $\mathrm{H}$ & $\mathrm{H}$ & $\mathrm{C}_{32} \mathrm{H}_{27} \mathrm{~N}_{3} \mathrm{O}_{2}$ & 485.58 & 108 & 0.64 \\
\hline 9 & APC-9 & $\mathrm{H}$ & $\mathrm{H}$ & $\mathrm{OCH}_{3}$ & $\mathrm{C}_{32} \mathrm{H}_{27} \mathrm{~N}_{3} \mathrm{O}_{3}$ & 501.58 & $138-140$ & 0.60 \\
\hline 10 & APC-10 & $\mathrm{H}$ & $\mathrm{H}$ & $\mathrm{NO}_{2}$ & $\mathrm{C}_{31} \mathrm{H}_{24} \mathrm{~N}_{4} \mathrm{O}_{4}$ & 516.55 & 112 & 0.50 \\
\hline 11 & APC-11 & $\mathrm{H}$ & $\mathrm{H}$ & ${\mathrm{N}\left(\mathrm{CH}_{3}\right)_{2}}$ & $\mathrm{C}_{33} \mathrm{H}_{30} \mathrm{~N}_{4} \mathrm{O}_{2}$ & 514.62 & $136-138$ & 0.44 \\
\hline 12 & APC-12 & $\mathrm{H}$ & $\mathrm{OCH}$ & $\mathrm{OCH}_{3}$ & $\mathrm{C}_{33} \mathrm{H}_{29} \mathrm{~N}_{3} \mathrm{O}_{4}$ & 531.60 & 148 & 0.51 \\
\hline
\end{tabular}

\section{Biological Evaluation}

Selected synthesized compounds were screened for their in vitro antibacterial and antifungal activity using representative strains of Gram-negative bacteria (Escherichia Coli, Pseudomonas Aeruginosa) and Gram-positive bacteria (Staphylococcus Aureus, Streptococcus Pyogenus). For antifungal activity, Candida Albicans, Aspergillus Niger and Aspergillus Clavatus were used as representative stains. The Ampicillin, Chloramphenicol, Ciprofloxacin and Norfloxacin were used as standard antibacterial drugs for the comparison, while, Nystatin and Greseofulvin were used as standard antifungal drugs. The broth dilution test method was followed for screening antimicrobial activity.

\section{Results and discussion:}

The present study describes the synthesis of twelve novel acetyl pyrazoline having biphenyl carbonitrile motif. The presence of characteristic peaks at 1657 and $1259 \mathrm{~cm}^{-1}$ in FT-IR confirmed the presence of carbonyl group. The characteristic bands present at $1604 \mathrm{~cm}^{-1}$ support the presence of pyrazoline ring. The characteristic peak observed at $2223 \mathrm{~cm}^{-1}$ confirmed the presence of nitrile group. Further, the presence of characteristic peaks observed in ${ }^{1} \mathrm{H}-\mathrm{NMR}$ and ${ }^{13} \mathrm{C}-\mathrm{NMR}$ confirms the structure of synthesized compounds.

(1) Antibacterial evaluation: Compounds APC-1, APC-10 and APC-11 are found equipotent to Ampicillin ( $\mathrm{MIC}=100 \mu \mathrm{g} / \mathrm{mL}$ ) against E.Coli. APC-12 is found equipotent to Chloramphenicol against P.Aeruginosa (gram -ve). Compounds APC-1, 2, 4, 5, 7, 9, 10 and 12 possess reasonably good antibacterial activity against S.Aureus (gram $+v e$ ) and found more efficient than Ampicillin $(\mathrm{MIC}=250 \mu \mathrm{g} / \mathrm{mL})$ in some cases. Compound APC-10 is found to equipotent to Chloramphenicol against S.Aureus. Compounds APC-5 is found to possess equipotent activity to Chloramphenicol against against $S$.Pyogenus (gram $+v e$ ). 
(2) Antifungal evaluation: Compounds APC-1, 2, 7, 9 and 10 are found equipotent to Greseofulvin $(\mathrm{MIC}=500 \mu \mathrm{g} / \mathrm{mL}$ ) against C.Albicans. While, all other synthesized compounds are less potent than standard drugs.

Table 1.2: Antibacterial Activity, Minimum Inhibition Concentration. (MIC ${ }^{\mathrm{a}}$ )

\begin{tabular}{|c|c|c|c|c|}
\hline \multirow{2}{*}{$\begin{array}{l}\text { Compound } \\
\text { Name }\end{array}$} & $\begin{array}{l}\text { E. Coli } \\
\text { (MIC)a }\end{array}$ & $\begin{array}{l}\text { P. Aeruginosa } \\
\text { (MIC)a }\end{array}$ & $\begin{array}{l}\text { S.Aureus } \\
\text { (MIC)a }\end{array}$ & $\begin{array}{l}\text { S.Pyogenus } \\
\text { (MIC)a }\end{array}$ \\
\hline & $\begin{array}{c}\text { MTCC } 443 \\
\text { Gram-Negative }\end{array}$ & $\begin{array}{c}\text { MTCC } 441 \\
\text { Gram-Negative }\end{array}$ & $\begin{array}{c}\text { MTCC } 96 \\
\text { Gram Positive }\end{array}$ & $\begin{array}{l}\text { MTCC } 442 \\
\text { Gram-Positive }\end{array}$ \\
\hline APC-1 & 100 & 250 & 200 & 250 \\
\hline APC-2 & 125 & 100 & 250 & 200 \\
\hline APC-4 & 500 & 250 & 125 & 100 \\
\hline APC-5 & 200 & 250 & 62.5 & 50 \\
\hline APC-6 & 500 & 500 & 500 & 250 \\
\hline APC-7 & 125 & 250 & 100 & 250 \\
\hline APC-9 & 125 & 100 & 125 & 125 \\
\hline APC-10 & 100 & 250 & 50 & 125 \\
\hline APC-11 & 62.5 & 100 & 500 & 500 \\
\hline APC-12 & 125 & 50 & 250 & 125 \\
\hline \multicolumn{5}{|l|}{ Standard drugs } \\
\hline Ampicillin & 100 & - & 250 & 100 \\
\hline Chloramphenicol & 50 & 50 & 50 & 50 \\
\hline Ciprofloxacin & 25 & 25 & 50 & 50 \\
\hline Norfloxacin & 10 & 10 & 10 & 10 \\
\hline
\end{tabular}

(MIC) $)^{\mathrm{a}}$ : Minimum Inhibitory concentration in $\mu \mathrm{g} / \mathrm{ml}$

Table 1.3: Antifungal activity, Minimum Fungicidal Concentration

\begin{tabular}{|c|c|c|c|}
\hline \multirow{2}{*}{ Compound Name } & $\begin{array}{c}\text { C.Albicans } \\
\text { (MIC)b }\end{array}$ & $\begin{array}{c}\text { A.Niger } \\
\text { (MIC)b }\end{array}$ & $\begin{array}{c}\text { A.Clavatus } \\
\text { (MIC)b }\end{array}$ \\
\cline { 2 - 4 } & MTCC 227 & MTCC 282 & MTCC 1323 \\
\hline APC-1 & $\mathbf{5 0 0}$ & 500 & 1000 \\
\hline APC-2 & $\mathbf{5 0 0}$ & 250 & 250 \\
\hline APC-4 & 1000 & 250 & 250 \\
\hline APC-5 & 1000 & 1000 & 1000 \\
\hline APC-6 & 1000 & 1000 & 1000 \\
\hline APC-7 & $\mathbf{5 0 0}$ & 1000 & 1000 \\
\hline APC-9 & $\mathbf{5 0 0}$ & 1000 & 500 \\
\hline APC-10 & $\mathbf{2 5 0}$ & 500 & 1000 \\
\hline APC-11 & 1000 & 500 & 1000 \\
\hline APC-12 & 1000 & 500 & 100 \\
\hline Standard drugs & & & 100 \\
\hline Nystatin & 100 & 100 & \\
\hline Greseofulvin & 500 & 100 & \\
\hline
\end{tabular}

(MIC) $)^{b}$ : Minimum Inhibitory concentration in $\mu \mathrm{g} / \mathrm{ml}$

\section{Conclusion:}

Total twelve derivatives of substituted acetyl pyrazoline having biphenyl carbonitrile motif has been synthesized in reasonably good yield. The spectral analysis; FT-IR, ${ }^{1} \mathrm{H}-\mathrm{NMR},{ }^{13} \mathrm{C}-\mathrm{NMR}$ and Mass spectral data confirmed the structures of synthesized compounds. Synthesized compounds were evaluated for biological property. Compounds APC-1, APC-2, APC-4, APC-5, APC-9, APC- 
10, APC-11 and APC-12 found to possess equipotent antimicrobial activity to standard drugs Ampicillin. Compounds APC-1, APC-2, APC-7, APC-9 and APC-10 found to posses comparable antifungal activity to standard drug Greseofulvin.

\section{Acknowldegement:}

Authors are thankful to SAIF and CIL, Punjab University for extending their support for instrumental analysis. Authors are also thankful to $\mathrm{M} / \mathrm{s}$ Solaris Chemtech Industries Pvt. Ltd, Baroda for providing 4'-bromomethyl-biphenyl-2-carbonitrile for this research work.

\section{References:}

[1] V.V. Kolta, V.K. Dalavai, V.R. Chanduri, Synthesis and biological activity studies of some novel pyrazoline derivatives, Der Pharma Chemica. 4(5) (2012) 2003-2008.

[2] M. Reddy et al., Synthesis, characterization and antimicrobial Activity of certain novel aryl hydrazone pyrazoline-5-ones containing thiazole moiety, Advanced Pharm. Bull. 3(1) (2013) 153-159.

[3] N.V Kavitha et al., Synthesis and antimicrobial activities of some new pyrazole derivatives, Der Pharma Chemica. 3(4) (2011) 55-62.

[4] M. Amir, H. Kumar, S. Khan, Synthesis and pharmacological evaluation of pyrazoline derivatives as new anti-inflammatory and analgesic agents, Bioorg. and Med. Chem. Lett. 18 (2008) 918-922.

[5] S.M. El-Moghazy, F.F. Barsoum, H.M. Abdel-Rahman, A.A. Marzouk, Synthesis and antiinflammatory activity of some pyrazole derivatives, Med. Chem. Res. 21 (2012) 1722-1733.

[6] M. Karabacak et al., Synthesis and evaluation of new pyrazoline derivatives as potential anticancer agents, Molecules. 20 (2015) 19066-19084.

[7] M. Shaharyar, M.M. Abdullah, M.A. Bakht, J. Majeed, Pyrazoline bearing benzimidazoles: Search for anticancer agent, Eur. J. Med. Chem. 45 (2010) 114-119.

[8] S. Gok, M. Demet, A. Ozdemir, G. Zitouni, Evaluation of antidepressant-like effect of 2pyrazoline derivatives, Med. Chem. Res. 19 (2010) 94-101.

[9] M.A. Ali, M. Shaharyar, A. A Siddiqui, Synthesis, structural activity relationship and antitubercular activity of novel pyrazoline derivatives, Eur. J. Med. Chem. 42(2) (2007) 268-275.

[10] P.T. Chovatia et al., Synthesis and selective antitubercular and antimicrobial inhibitory activity of 1-acetyl-3,5-diphenyl-4,5-dihydro-(1H)-pyrazole derivatives, J. Serb. Chem. Soc. 71(7) (2007) 713-720.

[11] F. Hayat, A. Salahuddin, S. Umar, A. Azam, Synthesis, characterization, antiamoebic activity and cytotoxicity of novel series of pyrazoline derivatives bearing quinoline tail, Eur. J. of Med. Chem. 45 (2010) 4669-4675.

[12] D.M. Martins et al., Antioxidant potential of new pyrazoline derivatives to prevent oxidative damage., Basic and Clin. Pharmacol. and Toxicol. 104(2) (2009) 107-112.

[13] V. H. Babu, C. Sridevi, A. Joseph, K. K. Srinivasan, Synthesis and biological evaluation of some novel pyrazolines, Ind. J. Pharm. Sci. 69 (2008) 470-473.

[14] S. Ovais et al., Synthesis and biological evaluation of some new pyrazoline substituted benzenesulfonylurea/thiourea derivatives as anti- hyperglycemic agents and aldose reductase inhibitors, Eur. J. Med. Chem. 80 (2014) 209-217.

[15] S. Ozdemir et al., Synthesis and studies on antidepressant and anticonvulsant activities of some 3-(2-furyl)-pyrazoline derivatives, Eur. J. Med. Chem. 42 (2007) 373-379. 
[16] S.G. Kucukguzel et al., Synthesis, characterization and pharmacological properties of some 4arylhydrazono-2-pyrazoline-5-one derivatives obtained from heterocyclic amines. Eur. J. Med. Chem. 35 (2000) 761-771.

[17] F. Chimenti et al., Synthesis, molecular modeling studies and selective inhibitory activity against MAO of N1-propanoyl-3,5-diphenyl-4,5-dihydro-(1H)-pyrazole derivatives, Eur. J. Med. Chem. 43 (2008) 2262-2267.

[18] Infectious Society of America, Statement of the IDSA Concerning "Bioshield II: Responding to a Diseases Ever-Changing Threat", IDSA, Alexandria, Va, USA, 2004.

[19] J.S. Bradley, R. Guidos, S. Baragona, Anti-infective research and development-problems, challenges and solutions, The Lancet Infectious Diseases. 7(1) (2007) 68-78.

[20] P.N. Patel, D.C. Karia, Synthesis and bioactivity evaluation of novel biphenyl thioxo pyrimidines as potent antimicrobial agent, Der Pharma Chemica. 7 (2015) 427-433. 